\title{
Hantavirus treatments advance amidst outbreak in US park
}

The often fatal lung disease caused by New World hantaviruses is rare. But, as a recent outbreak of the rodent-borne illness among visitors to California's Yosemite National Park has underscored, treatment is no walk in the woods. There are currently no specific medical countermeasures designed to combat the viral infection. And although early medical attention can increase the probability that someone with so-called 'hantavirus cardiopulmonary syndrome' (HCPS) will survive, around a third of all people with HCPS ultimately succumb to the disease.

"Even though it's not that frequent, it's a disease that's very catastrophic," says Pablo Vial, an infectious disease physician at the University for Development in Santiago, Chile, who treats people with HCPS. "Every time we have a case, we're seeing this young, healthy person with his whole future in front of him who was targeted by this virus-and we need to develop something for him."

Nobody appreciates that more than the US government, which remains one of the biggest supporters of HCPS research. That's in large part due to the numbers: with just over 600 documented cases of HCPS nationally since scientists first identified the virus in Arizona in 1993, the US-along with Argentina, Brazil and Chile-remains one of the countries hardest hit by the disease. (Hantaviruses found in Asia and Europe are responsible for a more common and less lethal disease known as 'hemorrhagic fever with renal syndrome', but only hantavirus strains of the New World can cause HCPS.) Another reason the US is heavily involved in developing antihantavirus therapeutics is logistics: much of the research on the lethal pathogens is restricted to the highest category of biosafety level laboratories, and all but one of these labs that study hantavirus are found in the US.

The research might be paying off. Christina Spiropoulou and her colleagues at the Viral Special Pathogens Branch of the US Centers for Disease Control and Prevention in Atlanta have been testing a number of small-molecule drugs for their diseasemitigating effects. The most promising seems to be an agent that blocks the vascular endothelial growth factor (VEGF) receptor. Two years ago, Spiropoulou's team demonstrated that VEGF signaling contributes to the breakdown of the endothelial barrier in hantavirus-infected human cell lines (J. Virol. 84, 11227-
11234, 2010). Now, the researchers have unpublished data showing that treatment with vandetanib, an antithyroid cancer drug marketed by the company AstraZeneca as Caprelsa that inhibits the VEGF receptor, can reduce vascular leakage and improve survival in hamsters. The effect was small, but "at least we know that we're in the right direction and there is a potential for this kind of treatment for HCPS," Spiropoulou says.

Vaccines also have potential. One of the most promising new therapeutics for HCPS was published earlier this year by Jay Hooper and his colleagues at the US Army Medical Research Institute of Infectious Diseases (USAMRIID) in Fort Detrick, Maryland. Reporting in PLoS One (7, e35996, 2012), the researchers showed that antibodies purified from the eggs of ducks that had been immunized with a DNA vaccine protected hamsters from lethal disease when given up to eight days after infection with the Andes hantavirus common to Chile and Argentina. In a similar vein, a team led by Heinz Feldmann, a virologist at the US National Institute of Allergy and Infectious Diseases Rocky Mountain Laboratories in Hamilton, Montana, reported late last year that hamsters vaccinated with an attenuated viral vector survived an otherwise lethal dose of the same hantavirus strain when challenged either before infection or up to 24 hours afterward (J. Virol. 85, 12781-12791, 2011).

\section{Outside the window}

An immunization strategy could be useful for military personnel who sleep on the ground in disease-endemic areas and individuals, such as those who stayed in the tent cabins in Yosemite, who might have unwittingly inhaled microscopic particles of urine or feces from hantavirus-infested deer mice. However, on the basis of the animal data, experimental vaccines such as those from Hooper's and Feldmann's labs might not offer relief for people arriving at the hospital already complaining of HCPS. "If you can detect [the virus] in the bloodstream, it's too late," Hooper says. "There's a pretty big window there, but once you're beyond the window we haven't seen a benefit [in hamsters]."

Pablo Vial has had some success treating people with symptoms of the disease. In an open-label trial that wrapped up earlier this year, Vial treated 35 people with fresh blood plasma taken from HCPS survivors that was rich in natural neutralizing antibodies. Only $13 \%$ of participants died, a level far below the $35 \%$ mortality rate normally seen in people infected with hantavirus. "Even though it's a very small experiment, we should go further following this approach," Vial says. He admits, however, that "the way to scale this up is difficult."

Back in the high-security labs in North America, most researchers continue to study hantaviruses in hamsters, which remain the only animal disease model for HCPS. Hantaviruses can infect other species, including macaque monkeys, but these animals generally remain symptom free. Only the Syrian hamster, in a model developed by Hooper a decade ago, develops a HCPS-like disease with its signature long incubation period and a rapid onset of severe respiratory illness. "This hamster model, if you look at the disease development, is as good as it can get to recapitulate the human situation," Feldmann says.

Still, the hamsters are far from perfect. Notably, they only succumb to the Andes hantavirus found mainly in Chile and Argentina, not the Sin Nombre strain responsible for most human deaths in the US. That's a problem for Hooper and Feldmann, who both have Sin Nombrespecific versions of their experimental vaccines but no disease model in which to test them. Additionally, with no alternate animal model available, even for the Andes hantavirus, Feldmann worries about how he can get his product cleared by the US Food and Drug Administration even under the agency's 'animal efficacy rule'. That rule provides a path to drug approval for lifethreatening agents where human efficacy trials aren't ethical or feasible but usually requires response data from more than one species. "We're desperately looking for a second animal model in this field," Feldmann says.

Elie Dolgin
Correction
In the September 2012 issue, the article entitled "Strapped for funding, medical researchers pitch to the crowd" (Nat. Med. 18, 1307, 2012) incorrectly listed Stephen Glatt's professional credentials. He's a psychologist, not a psychiatrist. The error has been corrected in the HTML and PDF versions of the article. 